\title{
Oliver Schöller
}

\section{"Geistige Orientierung" der Bertelsmann-Stiftung Beiträge einer deutschen Denkfabrik zur gesellschaftlichen Konstruktion der Wirklichkeit}

Auf dem informellen Jahrestreffen der international führenden MedienvertreterInnen im Oktober 1999 in Paris hielt der Soziologe Pierre Bourdieu die Eröffnungsrede. Nach eigener Aussage sprach er dort zu einer besonderen Art von Funktionselite, welche die vormals gesellschaftlich getrennten politischen, ökonomischen und symbolischen Machtressourcen heute in sich vereint (Bourdieu 1999). Diese Machtbündelung durch weltweit operierende Medienkonzerne wird kaum noch bestritten, allein die gesellschaftlichen Auswirkungen dieser Entwicklung werden kontrovers diskutiert. So hält beispielsweise Bourdieu den Befürwortern eines freien Medienmarktes, der ihrer Meinung nach durch seine Angebots- und Nachfrageregulierung auf gleichsam demokratische Weise für ein vielfältiges Angebot sorgt, die weltweit sich vollziehende „Uniformierung“ der Medienlandschaft entgegen.

Die von Bourdieu beschriebene weltweite Machtkonzentration im Feld der Massenmedien wird freilich erst vor dem Hintergrund des sich seit zwanzig Jahren vollziehenden tiefgreifenden technisch-ökonomischen Strukturwandels der Medienwirtschaft verständlich. Nachdem in den 80er Jahren die technischen Voraussetzungen für eine vielfältige und kostengünstige Distribution über Kabel und Satelliten geschaffen worden waren, begann der Kampf um das eigentlich knappe Gut im globalen Oligopol, die mediale ,Software': das Programm (Prokop 1995: 338). Im Verlaufe der daraufhin einsetzenden weltweiten Konzentrationsprozesse hatte sich die deutsche Bertelsmann-Gruppe schon Mitte der 80er Jahre als eines der weltweit größten multinationalen Unternehmen behauptet.

Auffallend ist allerdings, dass die in den letzten zwanzig Jahren zunehmende Konzentration in der Kommunikationsindustrie wissenschaftlich relativ wenig untersucht wurde. Diese Tatsache ist selbst wieder Ausdruck der Ungleichverteilung gesellschaftlicher Machtressourcen. So hat jüngst der Medienwissenschaftler Horst Röper festgestellt, dass es schon seit Jahren kaum mehr mög- 
lich ist, die zahlreichen Aktivitäten der deutschen Medienmultis umfassend zu schildern (Röper 1999). Den GesellschaftswissenschaftlerInnen fehlt es heute nicht zuletzt an den materiellen Mitteln, die für eine umfassende Analyse der Medienlandschaft, z.B. der nur schwer überschaubaren BertelsmannGruppe, nötig wären. Dabei hatte Jörg Becker schon frühzeitig auf ein Defizit der Untersuchung des Bertelsmann-Konzerns hingewiesen (Becker 1985). Gleichwohl gibt es bis heute keine umfassende kritische Studie über das Bertelsmann-Unternehmen. Dem stehen aber sehr viele Publikationen über die neuen Informations- und Kommunikationsmedien gegenüber, die von der dem Bertelsmann Konzern zugehörigen Bertelsmann-Stiftung herausgebracht wurden (vgl. Verlag Bertelsmann-Stiftung 1998/1999). Begreift man somit die allseits beklagte „Neue Unübersichtlichkeit“ (Habermas) nicht in erster Linie als das Resultat von Überkomplexität durch zunehmendes Wissen (Demirovic 1998: 63), sondern als das Ergebnis von Informationsdefiziten, die sich aus einer Ungleichverteilung gesellschaftlicher Machtpositionen ergeben, so erscheint eine eingehende Beschäftigung mit dem Medienkonzern Bertelsmann um so dringender. In dem vorliegenden Aufsatz werde ich mich nach einer kurzen Darstellung der wirtschaftlichen Aktivitäten des Bertelsmann Konzerns und daraus resultierender politischer Konsequenzen auf die Analyse der Ideologieproduktion bei der Konzernstiftung konzentrieren, die heute eine der größten „Denkfabriken“ der Bundesrepublik darstellt.

\section{Medienökonomie}

Die Fusionswellen in der Kommunikationsbranche haben in den letzten Jahren zusätzlich an Dynamik gewonnen. In Deutschland lassen sich die marktbeherrschenden Unternehmen in allen Mediensparten (Verlagswesen, Zeitungen, Funk und Fernsehen, Elektronische Medien) mittlerweile jeweils an einer Hand abzählen (Hautsch 1999).

Das Ziel der Konzentrationsprozesse besteht in der Schaffung ganzer Wertschöpfungsketten, wodurch sich einzelne Unternehmen z.B. sowohl den Einfluss über Fernsehsender sichern, wie auch die Filmproduktion und den Filmund Rechtehandel. Die im letzten Jahr vollzogene weltgrößte Fusion im Medienbereich von Time Warner, dem Internetanbieter AOL und dem Musikkonzern Emi machte diese Entwicklung erneut deutlich und gab ihr zugleich eine neue Qualität. So kann das Fernseh- und Zeitschriftenunternehmen Time Warner zukünftig seine Inhalte aus den Medien-, Unterhaltungs- und Nachrichtensparten zusätzlich über das Internet vermarkten. Das erste „Cross-Promoting“ dieser Art und Größenordnung praktizierte der Konzern jüngst bei der Vermarktung des neuen Buches von Jack Welch, dem Chef des Weltkonzerns General Electrics. Warner hatte die Konkurrenz - u.a. Bertelsmann - ausgestochen, indem das Unternehmen anbot, für das Buch nicht 
nur über $A O L$ und den Fernsehsender $C N N$ zu werben, ganz zu schweigen von dem hauseigenen Wirtschaftsmagazin Fortune, dem Time Magazin bis hin zum Klatschblatt People, sondern das Buch darüber hinaus auch in den Massenketten Walmart und Homedepot zu verkaufen (Fankfurter Rundschau 25.07.00).

Schließlich bringt die Fusion von Time Warner und AOL die zunehmende Bedeutung der Börsennotierung von Medienunternehmen zum Ausdruck. Waren bis Ende 1997 von deutschen Firmen allein die Pro Sieben Media AG und Bertelsmann börsennotiert, so sind mittlerweile über 16 weitere Firmen hinzugekommen (vgl. Frankfurter Allgemeine Zeitung, 29.06.99). Damit werden auch in der Medienbranche, vermittelt über das „Shareholder-ValuePrinzip“, die Verwertungsinteressen der Aktionäre zukünftig die Geschäftspolitik der Medienunternehmen maßgeblich mitbestimmen (Bischoff 2000). Auf diese Weise ist es möglich, dass der nach Umsätzen fünfmal kleinere Internetanbieter AOL mit seiner hohen Börsenbewertung den weltweit größten konventionellen Medienkonzern Time Warner übernehmen kann.

Auf dem deutschen Markt nimmt die Bertelsmann Gruppe seit Mitte der 80er Jahre mit Abstand die Spitzenposition ein. Aber auch international ist der Medienkonzern seitdem führend. Mit einem Gesamtumsatz von 6,2 Mrd. DM und fast 17.000 MitarbeiterInnen handelte es sich bei Bertelsmann 1985 um das weltweit größte multinationale Unternehmen, das ausschließlich im medialen ,Software'-Bereich tätig war (Becker 1985). Damals schon erwirtschaftete der Konzern über 50\% seiner Umsätze im Ausland. Umsatz und MitarbeiterInnenzahlen haben sich seitdem mehr als vervierfacht (Geschäftsbericht 1999/00). Noch bis Ende 1999 nahm der Bertelsmann Konzern auf der Liste der 50 größten Medienkonzerne der Welt den dritten Platz ein (vgl. Hachmeister/Rager 1997). Mit 14,8 Mrd. Dollar Jahresumsatz war Bertelsmann nach der Fusion von Viacom und CBS (18,9 Mrd.) im Herbst letzten Jahres, Disney/ABC (23 Mrd.) und Time Warner/AOL mit voraussichtlich $30 \mathrm{Mrd}$. Dollar Umsatz, zwischenzeitlich auf den vierten Platz zurückgefallen (Wirtschaftswoche 3/00). Jedoch zeigt der jüngste Bertelsmann-Coup im Februar diesen Jahres, dass der Bertelsmann Konzern auch in Zukunft an der Weltspitze mitmischen wird. Von der belgisch-kanadischen Investmentgesellschaft Group Bruxelles Lambert (GBL) erwarb das Medienunternehmen im Tausch gegen 25,1\% eigener Unternehmensanteile 30\% der Anteile der RTL-Gruppe. Damit konnte das Bertelsmann Unternehmen seine eigene Beteiligung an Europas größten Fernseh-, Radio- und Filmunternehmen auf 67\% erweitern und sich den beherrschenden Einfluss in diesem Marktsegment sichern. Die RTLGruppe war erst im April letzten Jahres durch die Fusion von CLT-Ufa und Pearson TV entstanden und vereint 160 Fernsehproduktionen in 35 Ländern, die mit 22 Fernsehsendern und 18 Radiostationen vertrieben werden. Analysten sehen in der strategischen Entscheidung des Bertelsmann Konzerns auch 
eine neue Gewichtung zugunsten der Offline-Medien, dem gegenüber den Online-Medien bedeutenderen Glied innerhalb der Wertschöpfungskette (vgl. taz vom 6.2.2001). Schließlich produzieren allein die Offline-Medien jene zur Vermarktung entscheidenden „Inhalte“ (Contents) und „Markennamen“ (Brands), welche daraufhin erst in einem zweiten Schritt auch über die Online-Medien vertrieben werden. „RTL als Portal in die Bertelsmann-Welt. Ein Marketinginstrument ohnegleichen - für viele Bertelsmann-Produkte“" (Frankfurter Rundschau 6.2.2001). Für den Bertelsmann Konzern beginnt zudem eine neue „Ära“ (Frankfurter Allgemeine Zeitung 6.2.2001). Bis heute ist das Familienunternehmen nur über einige Tochterfirmen, darunter Lycos Europe und dem Internetbuchhändler barnesandnoble.com, an der Börse vertreten. Die Finanzholding GBL plant nun ihre Bertelsmann-Anteile in drei Jahren an die Börse zu bringen. Damit wird auch das Stiftungsunternehmen Bertelsmann, entgegen den Beteuerungen seines Patriarchen Reinhard Mohn, zunehmend den Gesetzen des „Shareholder Value“ gehorchen.

\section{Politische Konsequenzen der Medienkonzentration}

Während man bei der Beschreibung der Entwicklungslinien der sogenannten Informationsgesellschaft weitgehende Einigkeit erzielt, ist demgegenüber unklar, wie sich die Mediatisierung der Gesellschaft auswirkt (Schatz/Jarren/ Knaup 1997). Jene mit der Entwicklung zur Wissensgesellschaft anfänglich verbundenen Verheißungen wie mehr Demokratie und bürgerliche Freiheit, allseitige Rationalität, neue qualifizierte Arbeitsplätze, Medienvielfalt etc. sind mittlerweile durch kritische Einwände ergänzt worden:

„Was bestritten wird, sind die umwälzenden Auswirkungen, die ihr [der Informatisierung, O.Sch.] mit der Theoriebildung um die ,Informationsgesellschaft' zugeschrieben werden. Die Gesellschaft wird diese Entwicklung,weg stecken', wie sie das Telefon und die modernen Verkehrstechniken weggesteckt hat" (Briefs 2000: 92).

Diese unaufgeregte Sicht der Dinge ist bei der sachlichen Medienanalyse zweifellos von Vorteil und ermöglicht z.B., wie dies jüngst Ingo Schmidt (2000) gezeigt hat, eine differenzierte Beurteilung der sich insbesondere im Bereich der IuK-Technologie vollziehenden Monopolisierungstendenzen. Sie sollte gleichwohl nicht zu der Annahme verleiten, alles bleibe beim Alten. Hat auch der Rückzug auf die nüchterne Beschreibung der Massenmedien einen Wert, so zeigt doch das in den 90er Jahren aufgekommene Interesse an gesellschaftlichen Macht- und Herrschaftsstrukturen die Relevanz von Ansätzen, welche sowohl das organisatorische Umfeld der Medieninstitutionen und ihre strukturellen Gegebenheiten als auch den Inhalt der Botschaften in ihrem Verhältnis zu den entsprechenden sozialen Kontexten berücksichtigen (Kannapin 1995). Auf diese Weise geraten die politischen und sozialen Folgen der Medienentwicklung in den Blick (Jäckel/Winterhoff-Spurk 1996). Deutlich wird sowohl der problematische Aspekt eines engen Beziehungsge- 
flechts von Politik und Medien (Hoffmann-Riem 2000), wie auch der ambivalente Charakter der Informationsgesellschaft selbst.

„Die Informationsgesellschaft kann Ungleichheiten verstärken, die in der Gesellschaft anderweitig verankert sind, nämlich in strukturell benachteiligten Lebenssituationen, die zum Beispiel durch Alter, Bildung, Lebensort, Armut, ethnische Herkunft und ähnliches geprägt sind“ (HoffmannRiem 1995: 29).

Gleichwohl gibt es von politischer Seite kaum Anzeichen, die sich abzeichnenden publizistischen Machtballungen durch regulierende Maßnahmen aktiv zu begleiten, „vielmehr scheint sich eine Tendenz zum weitgehenden laisser faire weiterzuentwickeln“ (Röper 1999: 345).

Die Berücksichtigung von Macht- und Herrschaftsstrukturen verdeutlicht aber auch den bedeutenden Einfluss von Medienkonzernen wie Bertelsmann auf die öffentlichen Diskurse. Wie schon des öfteren in der Vergangenheit demonstrierten die Bertelsmänner jüngst noch einmal, dass sie nicht nur die Macht besitzen darüber zu entscheiden, was die Öffentlichkeit lesen möchte, sondern auch was sie nicht lesen soll. ${ }^{1}$ Nachdem der Soziologe Hersch Fischler 1998 die vom Bertelsmann Konzern bis vor kurzem erfolgreich verschleierten Aktivitäten des Unternehmens während des Nationalsozialismus erforscht hatte, fand er in Deutschland kein Forum, in dem seine Ergebnisse publiziert wurden. Möglichkeiten der Veröffentlichung boten sich ihm schließlich in der Schweiz und den Vereinigten Staaten. ${ }^{2}$

\section{Tätigkeitsfelder und Ideologieproduktion der Bertelsmann-Stiftung}

Seit einigen Jahren wird auch in Deutschland zunehmend auf die gesellschaftspolitische Relevanz sog. Think Tanks hingewiesen (Gellner 1995, Reinicke 1996). Dabei hat man vor allem die lange US-amerikanische Tradition der über solche Institutionen vermittelten Politikberatung vor Augen. Diese hat in den 70er und 80er Jahren eine unter politischen Gesichtspunkten problematische Entwicklung durchgemacht. Während früher die gewählten VertreterInnen bei der Erarbeitung ihrer politischen Programmatik Expertengremien zeitweise zu Rate zogen, haben sich mittlerweile Planungsinstitute herausgebildet, die dauerhaft und relativ unabhängig von den eigentlichen demokratischen Institutionen politische Strategien formulieren. „Während politische Konzepte traditionellerweise durch die öffentliche Meinung und Parteienentscheidungen Eingang in die Agenda fanden, tauchen sie heute in

1 Vgl. die Liste öffentlicher Interventionen des Bertelsmann Konzerns in den 70er/80er Jahren bei Becker (1985), die zeigt, dass das Unternehmen schon damals immer dann mit Sanktionen reagierte, wenn es öffentlich in die Kritik geriet.

2 Vgl. die ausführliche Darstellung der Einflussnahme des Bertelsmann-Konzerns in der nationalen und internationalen Medienlandschaft, in: epd Medien, Nr. 17, 6. März 1999, ausgestellt unter www.epd.de/medien/1999. 
zunehmendem Maße ganz unabhängig von öffentlichen Debatten in den Institutionen der Politikberatung auf" (Fischer 1996: 470). In der zunehmenden Beeinflussung öffentlicher Diskurse durch private Institutionen liegt jedoch ein demokratietheoretisches Problem (vgl. Stone 1996). Es stellt sich die Frage, welche Perspektiven sich vor dem Hintergrund der US-amerikanischen Erfahrungen für die deutschen Verhältnisse ergeben.

Als eine der größten deutschen Stiftungen, die zudem aufgrund ihrer Nähe zu dem Medienkonzern Bertelsmann umfangreiche Ressourcen im Bereich der Printmedien nutzen kann, produziert die Bertelsmann-Stiftung seit Jahren zu nahezu allen gesellschaftlich relevanten Themen politische Strategiepapiere (vgl. Verlag Bertelsmann-Stiftung 1999/2000). Die Bertelsmann-Stiftung wurde 1977 von dem Patriarchen des Familienkonzerns Bertelsmann Reinhard Mohn gegründet. 1993 machte die Übertragung von rund 70\% der Kapitalanteile des Gesamtkonzerns auf die gemeinnützige Stiftung diese zum größten Kapitaleigner. ${ }^{3}$ Als Motiv für die Aufwertung der Bertelsmann-Stiftung gegenüber dem Konzern wird auf der Internetseite (www.stiftung. bertelsmann.de) die Überzeugung Mohns angeführt, „daß große Vermögen sich der Sozialverpflichtung des Eigentums, wie sie das deutsche Grundgesetz postuliert, unterzuordnen haben". Ob die Betonung dabei mehr auf der Sozialverpflichtung oder auf dem Eigentum liegt, soll hier nicht entschieden werden. Jedenfalls kommt die Stiftung im nächsten Absatz selbst auf die Steuervorteile zu sprechen, die ihr aus ihrer Rechtsform als gemeinnützige Stiftung des privaten Rechts laut Abgabenordnung zukommen. ${ }^{4}$ Ergänzend zur Bertelsmann-Stiftung arbeitet die 1995 von dem Bertelsmann Unternehmen gegründete Wissenschaftsstiftung. Die Bertelsmann-Stiftung beschäftigt heute über 130 MitarbeiterInnen, von denen wiederum über 130 Projekte betreut werden. Der Gesamtetat der Bertelsmann-Stiftung und der Bertelsmann Wissenschaftsstiftung stieg von 50 Mio. DM Mitte der 90er Jahre kontinuierlich bis auf 80 Mio. DM im Geschäftsjahr 1998/99.5

Die thematische Bandbreite der Bertelsmann-Stiftung umfasst die Bereiche Politik, Wirtschaft, Staat und Verwaltung, Hochschule, öffentliche Bibliotheken, Medien, Kultur und Medizin. Zur Bearbeitung dieser thematischen Viel-

3 Anteilseigner sind die Bertelsmann-Stiftung (68,9\% Kapital, kein Stimmrecht), die Bertelsmann Verwaltungs-GmbH (2,6\% Kapital, 89,3\% Stimmrecht; Vetorecht für Reinhard Mohn), die Familie Mohn (17,8\% Kapital, kein Stimmrecht) und die ZEIT-Stiftung (10,7\% Kapital, 10,7\% Stimmrecht; soll aufgekauft werden). Der Konzern ist an über 600 Firmen in 50 Ländern beteiligt (aus: Reader Medienökonomie 1999: 16).

4 An anderer Stelle gibt Mohn als weiteres Motiv für die Gründung einer gemeinnützigen Stiftung an, auf diese Weise die Erbschaftssteuer zu umgehen und damit die Unternehmenskontinuität zu sichern (Mohn 1997b: 25).

5 Die Bertelsmann-Stiftung ist nach Jahresausgaben mittlerweile Deutschlands drittgrößte Stiftung hinter der Deutschen Bundesstiftung Umwelt auf dem zweiten und der Volkswagen Stiftung auf dem ersten Platz (vgl. Bundesverband Deutsche Stiftungen 2000). 
falt hat die Stiftung in den 90er Jahren eine ganze Reihe von Forschungsgruppen, Kommissionen und Arbeitsgruppen gegründet, die kontinuierlich über längere Zeiträume gesellschaftspolitische Konzepte entwickeln und damit gezielt öffentliche Diskurse speisen (vgl. Tätigkeitsbericht 98/99). Die Bertelsmann-Stiftung hat keinerlei Berührungsängste hierbei Kooperationen mit den unterschiedlichsten Stiftungen einzugehen, darunter sowohl die Heinz Nixdorf und die Ludwig-Erhard-Stiftung wie auch die Heinrich-Böll und die Hans-Böckler-Stiftung.

Die einflussreiche Rolle der Bertelsmann-Stiftung verdeutlicht insbesondere die mittlerweile langjährige Zusammenarbeit mit der Stiftung des Deutschen Gewerkschaftsbunds, der Hans-Böckler-Stiftung. Zusammen haben sie seit 1996 in der sog. Kommission Mitbestimmung Ansätze für eine neue „Unternehmenskultur" entwickelt (vgl. Bertelsmann-Stiftung/Hans-Böckler-Stiftung 1998). Das gemeinsame Ziel besteht in dem Aufbau einer Vertrauenskultur auf Betriebsebene, die es ermöglichen soll, die neuen globalen Wettbewerbsbedingungen mit den Wünschen und Interessen der Mitarbeiter in Einklang zu bringen. Ein erster Zwischenbericht wurde dem Bundespräsidenten im Mai 1998 medienwirksam überreicht. Das in dem Zwischenbericht propagierte Konzept einer markt- und mitarbeiterorientierten Unternehmenskultur wird durch ein sechs Bände umfassendes, praxisorientiertes Handbuch verbreitet, das letztes Jahr in vollständig überarbeiteter und erweiterter Neuauflage erschien (Bertelsmann-Stiftung/Hans-Böckler-Stiftung 2000). Mittlerweile setzen beide Stiftungen ihre Tätigkeit in dem 1999 in Düsseldorf gegründeten Forum Mitbestimmung und Unternehmen fort. Freilich wurde mit Blick auf die gut untersuchte Unternehmenskultur des Bertelsmannkonzerns, welche sich durch autoritäre Strukturen auszeichnet (vgl. Bundesmann/Pekruhl 1992), schon des öfteren gefragt, ob eine Wirtschaftsstiftung wie die Bertelsmann-Stiftung der richtige Partner für eine Gewerkschaftsstiftung sei, der es darum gehen sollte, demokratische Formen der Mitbestimmung zu entwickeln (Wehner 1998; ders. 1999). Der problematische Charakter dieser Zusammenarbeit wird besonders vor dem Hintergrund der innerhalb der Gewerkschaften zur Zeit kontrovers diskutierten Novellierung des Betriebsverfassungsgesetzes von 1972 deutlich. Während die Vorschläge der „Kommission Mitbestimmung" demokratische Partizipationsmöglichkeiten im Wesentlichen als einen „harmonischen Anpassungsprozess“ an die neue ökonomische Funktionslogik diskutieren (vgl. Martens 1999; Deppe 2000), betont demgegenüber der Bundesvorstand des DGB den notwendigen Ausbau institutionalisierter und rechtlich fixierter Mitbestimmungsrechte, die auch gegen divergierende Unternehmensinteressen durchgesetzt werden können (vgl. DGB 1998). Der Einfluss, den die Bertelsmann-Stiftung über die Zusammenarbeit mit der Hans-BöcklerStiftung auf diese zentrale Debatte ausübt, ist mithin nicht zu unterschätzen und verdeutlicht schlaglichtartig ihre gesellschaftliche Definitionsmacht. 
Der Einflussbereich der Bertelsmann-Stiftung beschränkt sich jedoch nicht allein auf Stiftungskooperationen sondern erstreckt sich bis in staatliche Institutionen hinein. Ein Beispiel dafür sind ihre Aktivitäten im Bereich der Hochschulpolitik. Die Bertelsmann-Stiftung gründete zusammen mit der Hochschulrektorenkonferenz 1994 das „Centrum für Hochschulentwicklung“ (CHE). Das CHE beteiligt sich aktiv an den Hochschulreformdebatten und ist bestrebt, „dem politischen Meinungs- und Willensbildungsprozess frühzeitig Impulse zu geben und die öffentliche Diskussion zu bündeln“ (Tätigkeitsbericht 98/99: 90). Auch die bildungspolitischen Aktivitäten der Bertelsmann-Stiftung wurden in der Vergangenheit immer wieder vom damaligen Bundespräsidenten unterstützt. Unter seiner Schirmherrschaft etablierte das CHE den Initiativkreis Bildung der ebenfalls Vorschläge zur „Erneuerung des Bildungswesens“ erarbeitete (vgl. Memorandum 1999). Darüber hinaus wirkt die private Unternehmensstiftung vermittelt über das CHE auch direkt auf öffentliche Institutionen wie die Hochschulrektorenkonferenz ein. Über weitreichende personelle Verflechtungen wiederum ergeben sich Kontakte zur Hochschulstrukturkommission in Baden-Württemberg, dem Bund Norddeutscher Hochschulen, dem Ministerium für Wissenschaft, Forschung und Kultur des Landes Thüringen, sowie dem Ministerium für Schule und Weiterbildung, Wissenschaft und Forschung in Nordrhein-Westfalen. Der Rechtssoziologe Martin Bennhold fasst in seiner Studie über das CHE die Folgen prägnant zusammen:

„Es liegt auf der Hand, wie damit Zuständigkeitsbereiche, die öffentlicher Kontrolle zu unterliegen haben, von privaten Einrichtungen Schritt für Schritt besetzt werden sollen und wie dadurch das interessierte Diktat, die schiere Kontrolle durch das Kapital und seine Funktionäre, immer weitere Bereiche der Gesellschaft erfasst und die restlichen Entscheidungs- und Kontrollkompetenzen öffentlicher Organe vollends aushöhlt oder zu bloßen Instanzen der Legitimation entwertet" (Bennhold 1999: 54).

Deutlich wird, warum sich die Bertelsmann-Stiftung selbst als eine sog. „Operative Stiftung" versteht, die Projekte anregt und sie bis zu ihrer Umsetzung gestalterisch und organisatorisch begleitet. „Operative Stiftungsarbeit richtet sich dabei auf leistungsorientierte reformerische Lösungsstrategien für gesellschaftliche Probleme, welche die Stiftung selbst aufgreift" (BertelsmannStiftung 1997: 9). Mit ihrem eigenständigen agenda-setting knüpft die Bertelsmann-Stiftung explizit an die US-amerikanische Tradition unabhängiger Think Tanks an:

„Kleinere ebenso wie große Stiftungen und insbesondere die Think Tanks spielen in der Gestaltung der [US-amerikanischen; O. Sch.] Gesellschaft und in bezug auf die erforderliche Systementwicklung eine unverzichtbare Rolle. - Die Bertelsmann-Stiftung schlägt vor, aus diesen internationalen Erfahrungen zu lernen und zugleich dazu anzuregen, ähnliche Initiativen auch in unserem Land auszulösen“ (Mohn 1996c: 13).

Gleichwohl unterscheidet sich die Bertelsmann-Stiftung von ihren USamerikanischen Vorbildern dahingehend, dass sie, anders als die neoliberalen 
Denkfabriken in den Vereinigten Staaten, eine neokorporatistische Strategie verfolgt. Diese zeichnet sich insbesondere dadurch aus, dass, wie die Zusammenarbeit mit der Hans-Böckler-Stiftung zeigt, die Unternehmensstiftung von Anfang an bestrebt ist, die Gewerkschaften mit einzubeziehen. Zudem gelingt es der Stiftung unterschiedliche Personen und Institutionen mit zum Teil stark differierenden politischen Ausrichtungen in eine Vielzahl ihrer Projekte einzubinden.

Die Bertelsmann-Stiftung begründet die Notwendigkeit operativer Stiftungen mit der Unfähigkeit des Staates, sich zuspitzende gesellschaftliche Probleme einer Lösung zuzuführen (Bertelsmann-Stiftung 1997: 9). Neben einer in der Bertelsmann-Stiftung stark ausgeprägten einseitig anti-staatlichen Haltung, die insbesondere durch den Stiftungsgründer Mohn vertreten wird, propagiert die Stiftung das Ideal des auf dem freien Markt gereiften Unternehmensführers (vgl. Mohn 1997: 24). Charismatische Unternehmerpersönlichkeiten sollen daher die Leitung gemeinnütziger operativer Stiftungen übernehmen, um auf diese Weise als „Impulsgeber“ für gesellschaftsverändernde Initiativen zu wirken (ebd.: 31). Schließlich schreibt sich die Bertelsmann-Stiftung selbst eine zentrale Rolle als Vorreiter gesellschaftlicher Veränderungen zu (vgl. Bertelsmann-Stiftung 1997: 11). In Anbetracht dieser offensiven Strategie einer zweifellos definitionsmächtigen, eng an Unternehmensinteressen gebundenen Denkfabrik, die gesellschaftliche Veränderungen anstrebt, ohne dazu im traditionellen Sinne demokratisch legitimiert zu sein, interessiert in besonderem Maße die inhaltliche Ausrichtung jener programmatischen Konzepte, die im Auftrag der Bertelsmann-Stiftung produziert werden. So liegt beispielsweise die Frage nahe, ob die enge Verbindung von Bertelsmann Unternehmen und Stiftung, wie sie finanziell durch die schon beschriebene Kapitalbeteiligung und personell durch den Unternehmensinhaber und jetzigen Leiter der Stiftung Reinhard Mohn gegeben ist, einen Einfluss auf die gesellschaftspolitischen Studien der Stiftung hat. Folgt man Mohn selbst, der jüngst in einem Interview feststellte, dass der alte Interessenkonflikt von Kapital und Lohnarbeit unter den heutigen Bedingungen differenzierter Gesellschaften nicht mehr existiert (Mohn 1999), dann würde sich auch die Kritik an durch privatwirtschaftliche Interessen geleiteten und die Öffentlichkeit beherrschenden Diskursen erübrigen. Stattdessen soll im folgenden die Analyse des von der Bertelsmann-Stiftung ins Leben gerufenen Projekts „Geistige Orientierung“ Aufschluss über den Gehalt der durch die Stiftung geförderten Ideologieproduktion geben.

\section{Das Projekt „Geistige Orientierung" der Bertelsmann-Stiftung}

\section{A. Rahmenhandlung}

Es wäre wohl naiv, zwischen dem Bertelsmann Konzern und seiner Stiftung bewusste Formen der Manipulation nachweisen zu wollen. Ebenso wäre es 
missverständlich hier von Filz zu reden, bezeichnet man damit doch traditionell ein enges, für Außenstehende undurchschaubares Beziehungsgeflecht wechselseitiger Begünstigung, das sich als Machtverhältnis verselbständigt. Dieses Bild der Filzokratie wird jedoch den heutigen Verhältnissen hochgradig rational organisierter Unternehmen nicht gerecht (Bundesmann-Jansen/ Pekruhl 1992). Vielmehr sind die vielfältigen Stiftungsprojekte im gesamtgesellschaftlichen Kontext zu analysieren und ihre jeweilige Funktion als Beiträge zu politischen, ökonomischen sowie kulturellen Diskursen darzustellen.

Das Projekt „Geistige Orientierung“ der Bertelsmann-Stiftung ist auf die besondere Initiative ihres Leiters Reinhard Mohn zurückzuführen. Dieser hat die Bekämpfung geistiger Orientierungslosigkeit zu seinem persönlichen Anliegen gemacht. Seit Anfang der 90er Jahre äußert sich Mohn im Rahmen kleiner Broschüren, die kostenlos beim Verlag der Stiftung (auch in englischer Sprache) erhältlich sind und in denen er es auf jeweils durchschnittlich zwanzig Seiten unternimmt, prägnant Sinn zu stiften (Mohn 1992, 1992a, 1993, 1995, 1996, 1996a, 1996b, 1996c, 1997, 1997a, 1997b, 1998). Für Mohn zeichnen sich die modernen Gesellschaften durch einen allgemeinen Werte-Zerfall aus:

„Die heute täglich demonstrierte Unfähigkeit zum Konsens innerhalb unserer Gesellschaft beruht weniger auf unseren unzulänglichen Führungs- und Ordnungsstrukturen als auf einem weitgehenden Verlust gemeinsamer Werte und Ziele (Mohn 1997a: 5f.).“

Die Ursachen für den Werte-Zerfall und die daraus resultierende Orientierungslosigkeit der Gesellschaftsmitglieder sieht Mohn wesentlich in einer dekadenten Entwicklung der westlichen Industrienationen begründet. Diese habe in Deutschland nach dem Zweiten Weltkrieg mit dem „Wirtschaftswunder" eingesetzt und schließlich zu einer übertriebenen Selbstbezogenheit der Konsummenschen geführt, denen heute weitgehend jeglicher Sinn für Gemeinschaftsfähigkeit fehle.

„Unsere Gesellschaft erscheint aufgesplittert in Gleichgültige und Interessengruppen, die im falsch verstandenen Recht zur Selbstverwirklichung ihre Interessen rechthaberisch und rücksichtslos durchsetzen“ (Mohn 1997: 6).

In diesem Sinne veröffentlichte die Stiftung mit dem von Werner Weidenfeld $^{6}$ und Dirk Rumberg herausgegebenen Sammelband Orientierungsverlust - Zur Bindungskrise der modernen Gesellschaft (1994) eine Bestandsaufnahme und rief damit zugleich das Projekt „Geistige Orientierung“ ins Leben. Daraufhin wurden die Soziologen Peter Berger und Thomas Luckmann beauftragt, eine erste wissenschaftliche Analyse der Entwicklung moderner Gesellschaften zu erstellen, die 1995 unter dem Titel Modernität, Pluralismus und Sinnkrise. Die Orientierung des modernen Menschen erschien. Ebenfalls

6 Werner Weidenfeld ist neben Reinhart Mohn und Thomas Middelhof Vorstandsmitglied der Bertelsmann-Stiftung. 
im Rahmen des Projekts „Geistige Orientierung“ veröffentlichte die Bertelsmann-Stiftung die von Berger herausgegebene Studie Die Grenzen der Gemeinschaft. Konflikt und Vermittlung in pluralistischen Gesellschaften (1997). Dabei handelt es sich um eine weltweit vergleichende Untersuchung von elf Ländern, ${ }^{7}$ deren Ergebnisse von der Bertelsmann-Stiftung an den Club of Rome übergeben wurden. Der Bericht der Bertelsmann-Stiftung knüpft dabei mit dem Ziel öffentlicher Intervention ganz bewusst an die Strategie des Club of Rome an, doch anstatt die Knappheit materieller Ressourcen anzumahnen, verweist der Stiftungsbericht auf die Gefährdung der modernen Gesellschaften durch das Austrocknen kultureller Ressourcen:

„Wir müssen uns darüber klar werden, daß es nicht nur Grenzen des Wachstums gibt, sondern auch Grenzen der sozialen Kohäsion, von denen unser Überleben als Menschen unter friedfertigen gesellschaftlichen Bedingungen abhängt. Dieser Bericht will dem Club of Rome und der Öffentlichkeit die Botschaft vermitteln, daß wir alle mit den kulturellen Ressourcen unserer Gesellschaften, mit Normen, Werten und gesellschaftlichen Grundhaltungen auf sehr sensible und sorgfältige Weise umgehen müssen." (Weidenfeld 1997: 13)

Diese internationale Untersuchung wurde schließlich durch eine von Luckmann herausgegebene nationale Studie mit dem Titel Moral im Alltag. Sinnvermittlung und moralische Kommunikation in intermediären Institutionen (1998) ergänzt. Darüber hinaus hat die Stiftung parallel eine ganze Reihe von Einzelgutachten in Auftrag gegeben ${ }^{8}$, die im weiteren jedoch nur am Rande Erwähnung finden, da die wesentlichen Grundlagen, um deren Darstellung es hier geht, durch die Arbeiten von Berger und Luckmann gelegt wurden.

\section{B. Die gesellschaftliche Konstruktion der Wirklichkeit und die Sinnkrise moderner Gesellschaften nach Peter Berger und Thomas Luckmann}

Berger/Luckmann unternehmen in ihrer Abhandlung Modernität, Pluralismus und Sinnkrise den Versuch einer Erklärung der historisch spezifischen über Sinn vermittelten Vergesellschaftung in modernen Gesellschaften?. Bei ihren grundlegenden Ausführungen in den ersten beiden Kapiteln über menschliche Sinnhaftigkeit und Sinnproduktion in sozialen Beziehungen handelt es sich im wesentlichen um eine Zusammenfassung der Ergebnisse ihrer Theorie der Wissenssoziologie aus dem Jahre 1969 (Berger/Luckmann 1969). Ausgehend vom menschlichen Bewusstsein und seiner anthropologischen Fähigkeit der Sinnkonstituierung, entwickeln Berger/Luckmann ein komplexes Bild vom menschlichen Individuum in sozialen Beziehungen. Da

7 USA, Frankreich, Deutschland, Ungarn, Chile, Südafrika, Türkei, Indonesien, Indien, Japan, Taiwan.

8 Dettling (1995), Schmidtchen (1996), Greiffenhagen (1997), Bertram (1997), Huber (1998); in Arbeit sind Studien von Gerhard Schulze, Axel Honneth und Eckart Liebau.

9 Zur Zitierweise: Ist im folgenden keine andere Quelle angegeben, dann beziehen sich die Seitenangaben auf diesen Titel. 
sich menschliches Bewusstsein nur als bewusstes Sein konstituieren kann, setzt dies immer schon die bewusste Erfahrung der Umwelt voraus. Erst die Bezugnahme auf einen gesicherten Bestand akkumulierter Umwelterfahrungen ermöglicht die sinnhafte Kontextualisierung von Alltagserfahrungen. Damit ist „Sinn nichts anderes als eine komplexe Form von Bewusstsein: Er existiert nicht für sich allein, sondern hat immer ein Bezugsobjekt" (11). Persönliche Identität konstituiert sich mithin notwendigerweise als das Ergebnis über Handlungen vermittelter sozialer Beziehungen. Aber auch individuelle Handlungsmuster sind nur denkbar auf dem Hintergrund gesellschaftlich objektivierter Sinnstrukturen. In zunehmend ausdifferenzierten Gesellschaften bilden sich Berger/Luckmann zufolge schließlich Institutionen heraus, deren wesentliche Funktion in der Kontrolle der Sinnproduktion und der Sinnvermittlung besteht. Ausgehend von der Überzeugung, dass Lebensgemeinschaften grundsätzlich ein Mindestmaß an Sinngemeinsamkeit voraussetzen, entwerfen die Autoren das Bild einer Sinngemeinschaft, die sich durch ein individuell, kollektiv und institutionell konstruiertes Beziehungsgeflecht auszeichnet.

Berger/Luckmann zufolge ist der daraus resultierende Sinnkontext grundsätzlich durch subjektive und intersubjektive Sinnkrisen gefährdet. Besonders anfällig für strukturelle Sinnkrisen sind jedoch Gesellschaften, die sich durch ein Nebeneinander unterschiedlicher Sinngemeinschaften auszeichnen.

„Der Zustand, der sich aus diesen Voraussetzungen ergibt, kann als Pluralismus bezeichnet werden. Wenn er sich selbst zu einem übergreifenden Wert für die Gesellschaft entwickelt, können wir vom modernen Pluralismus sprechen“ (30).

Es ist aber gerade dieser moderne Pluralismus aus dem heraus die Autoren in den folgenden Kapiteln die aktuelle Orientierungslosigkeit der Menschen erklären, und wodurch zugleich die reibungslose Vergesellschaftung in Frage gestellt sei. Lehnen Berger/Luckmann es auch ab, von einer „umfassenden Sinnkrise“ (36) zu sprechen, so diagnostizieren sie gleichwohl eine weit verbreitete Unsicherheit „sowohl in der einzelnen Handlungs- wie in der gesamten Lebensorientierung" (ebd.).

Da Berger/Luckmann in dem von ihnen untersuchten gesamtgesellschaftlichen Phänomen der Orientierungslosigkeit offensichtlich vor allem ein Verständigungsproblem sehen, propagieren sie folgerichtig die Eindämmung überbordender Deutungsmöglichkeiten durch eine Artikulation kleiner Lebens- und Sinngemeinschaften, die sich jeweils durch einen wie auch immer gearteten „Zaun“ von den sie umgebenden Gruppen abgrenzen sollen, um für sich auf diese Weise neue Übersichtlichkeit zu schaffen:

„Wenn es nicht gelingt, die durch die Pluralisierung ermöglichte Interaktion durch ,Zäune der einen oder anderen Art zu beschränken, wird der Pluralismus voll wirksam und damit auch eine seiner Folgen: die ,strukturelle' Sinnkrise“ (42).

Orte der Sinnproduktion in modernen Gesellschaften, die es den „modernen Menschen“ ermöglichen, innerhalb (selbst-) definierter Grenzen sinnvoll zu 
leben, sind Berger/Luckmann zufolge sog. „intermediäre Institutionen“, die zwischen dem einzelnen und der Gesellschaft vermitteln (59). Die Entscheidung darüber, welche gesellschaftlichen Einrichtungen nun als intermediäre Institutionen fungieren, überlassen sie an dieser Stelle noch zukünftigen empirischen Untersuchungen, wenngleich sie andeuten, dass „eine kirchliche Ortsgemeinde, eine psychotherapeutische Gruppe, selbst eine Agentur des Wohlfahrtsstaates im Erleben der damit verbundenen Menschen eine wirklich vermittelnde, intermediäre Institution sein (kann)“ (59).

In der später von Luckmann (1998) herausgegebenen empirischen Studie werden die Familie und die Ökobewegung als „echte“ intermediäre Institutionen beschrieben, während sich demgegenüber die dort ebenfalls untersuchten Beratungseinrichtungen durch „Werturteilsabstinenz“ auszeichnen und damit keine ihrer Definition gemäße sinnstiftende Funktion übernehmen. Aber auch die Kirche ist mittlerweile in einer eigenen Studie als mögliche intermediäre Institution identifiziert worden (Huber 1998), ganz im Sinne Berger/Luckmanns, die ihr immer schon ,eine äußerst positive Funktion [...] in bezug auf das Leben des einzelnen wie das der Gesamtgesellschaft" (60) eingeräumt haben. Demgegenüber verweist Berger mittlerweile auf die Ergebnisse der von ihm herausgegebenen international vergleichenden Studie Die Grenzen der Gemeinschaft, wo sich gezeigt habe, dass die selben Institutionen bei normativen Konflikten in einem Fall polarisierend wirken, während sie in einem anderen Fall vermitteln. Deshalb mache es keinen Sinn, „eine Liste polarisierender Institutionen zu erarbeiten und sie einer Liste der intermediären Institutionen gegenüberzustellen" (Berger 1997: 596). Intermediäre Institutionen im Sinne Berger/Luckmanns können jedoch allgemein als Einrichtungen bezeichnet werden, die dem sog. „Dritten Sektor“ (vgl. Nielandt/ Brodkorb 2000, Salamon/Anheier 2000) zugerechnet werden, und die, organisatorisch und funktional verortet zwischen den ,großen Institutionen der Gesamtgesellschaft und dem einzelnen in seinen Lebensgemeinschaften“" (76), die Herausbildung zivilgesellschaftlicher Strukturen befördern.

Doch unabhängig davon, welche zivilgesellschaftlichen Organisationen nun tatsächlich in ihrer janusköpfigen Funktion als vermittelnde Instanzen der Sinnproduktion zwischen Individuum und Staat fungieren: die Massenmedien, so Berger/Luckmann, spielen bei der modernen Sinnorientierung bzw. Sinnverteilung eine Schlüsselrolle:

„Sie vermitteln zwischen kollektiver und individueller Erfahrung, indem sie typische Deutungen für als typisch definierte Probleme anbieten. Was immer andere Institutionen an Wirklichkeitsdeutungen und Werten produzieren, die Medien wählen aus, organisieren (,verpacken') diese Produkte, verändern sie meistens im Lauf dieser Prozesse und entscheiden über die Formen der Verbreitung" (57).

In Anbetracht der Bedeutung, die den Massenmedien hier ganz unvermittelt zugeschrieben werden, überrascht die ansonsten durchgehende Fixierung der 
Autoren auf die intermediären Institutionen. Es fällt auf, dass die gesellschaftliche Funktion der Massenmedien an keiner Stelle problematisiert wird. Im Gegenteil, mit Blick auf die intermediären Institutionen wird den Massenmedien eine ausschließlich positive Rolle zugeschrieben:

„Es liegt in der Verantwortung und, wie wir meinen, auch der konkreten Verwirklichungsmöglichkeit der Führungskräfte der Sinnvermittlungsanstalten, wie z.B. der Massenmedien, im Rahmen eines ,deregulierten' Sinnangebotsmarktes intermediäre Institutionen zu stützen“" (71).

Unterschiede zwischen privatwirtschaftlichen Medien und öffentlichen Anstalten finden Erwähnung (75), werden jedoch nicht ausgeführt. Stattdessen sehen Berger/Luckmann die intermediären Institutionen offensichtlich vor allem durch den Staat gefährdet: „Bezeichnenderweise ist es nicht selten der Staat, der an der Schwächung intermediärer Institutionen [...] mitwirkt" (63). Mit ihrer Stoßrichtung gegen wohlfahrtsstaatliche Einrichtungen folgen die Autoren ihrem Auftraggeber Reinhard Mohn, der ebenfalls den aufgeblähten Staatsapparat als den Ausdruck einer dekadenten Anspruchsmentalität sowie daraus resultierender Innovationsfeindlichkeit ausmacht (Mohn 1997a). Dementsprechend werden die zu beobachtenden krisenhaften Individualisierungsprozesse nicht etwa mit Bezug auf das kapitalistische Wirtschaftssystem analysiert, sondern kurzerhand als Folge des Wohlfahrtsstaats interpretiert. In der globalen Ökonomie hingegen verortet Mohn die Springquellen neuer Kreativität: „Der zunehmende Druck des globalen Wettbewerbs in der Wirtschaft scheint hier die ersten Reformmöglichkeiten herbeizuführen“" (Mohn 1997: 7). Auch von Berger/Luckmann werden Wirtschaft und Staat, Marktwirtschaft und Demokratie als die ,zentralen Institutionen der modernen Gesellschaft“ (50) nicht mehr kategorisch unterschieden sondern verkürzt auf die Gemeinsamkeit der Wahlmöglichkeit bzw. des Wahlzwangs. Widerstrebende Interessen zwischen der privaten und der öffentlichen Sphäre bürgerlich-kapitalistischer Gesellschaften erscheinen bei ihnen reduziert auf Konflikte unterschiedlicher Orientierungsangebote die über den „Markt“ reguliert werden und sich in verschiedenen Lebenszielen und -inhalten manifestieren (51).

Die hier artikulierte Position gegenüber den staatlichen Institutionen erinnert an jene der Kommunitaristen und deren Fixierung auf überschaubare Formen der Vergesellschaftung in Kleingruppen bzw. der Familie. Aber ebenso wie die Strategien des Kommunitarismus und des Neoliberalismus in ihrer verkürzten Staatskritik übereinstimmen, indem sie ausschließlich in einer AntiHaltung verharren und die Beschränkung der Staatsgewalt fordern, trifft dies auch für die Bertelsmann-Stiftung zu.

Es ist erstaunlich wie weit sich Berger/Luckmann bei ihrer Analyse der Sinnkonstituierung des „modernen Menschen” schließlich von dessen konkreten lebensweltlichen Verhältnissen und damit von den materiellen Voraussetzungen menschlicher Identitätsbildung entfernen. Dabei hatten sie eingangs noch die Bedeutung des historisch je spezifischen Erfahrungshorizonts bei 
der menschlichen Bewusstseinsbildung hervorgehoben. So stellen sie z.B. fest, dass der moderne Pluralismus oftmals recht unterschiedlich auf die Gesellschaftsmitglieder wirkt und nicht bei allen zur Verunsicherungen führt, ja es gebe sogar regelrechte „Virtuosen des Pluralismus“ (45). Dieses zweifellos interessante Phänomen wird nun aber nicht etwa zum Anlass weiterer Untersuchungen, schließlich könnte man in den Lebenswelten jener offenbar besonders resistenten Spezies nach den Gründen dafür forschen, dass sich diese unter den neuen Bedingungen wie Fische im Wasser fühlen, während andere ertrinken. So kamen empirische Untersuchungen schon in der Vergangenheit zu dem Ergebnis, dass es sich bei den Virtuosen des Pluralismus mit ihren „Patchwork-Identitäten“ und „Bastelbiographien“ (Keupp 1992: 176), um ein Phänomen der Mittelschichten handelt, während in den Unterschichten das Diktat der Notwendigkeiten und der Zwang der materiellen Verhältnisse kaum Spielraum für Stilisierungsexperimente lassen (Müller 1992: 375). Berger/Luckmann hingegen sehen in der Ausnahme lediglich die Bestätigung der Regel und verweisen auf die Mehrzahl desorientierter Menschen in modernen Gesellschaften (45).

Diese Realitätsferne schlägt sich schließlich ebenso in der Analyse zivilgesellschaftlicher Strukturen nieder. Das von Berger/Luckmann propagierte Projekt intermediärer Institutionen, welche die lebensweltliche Artikulation individueller Bedürfnisse gewährleisten soll, wird konterkariert durch die besondere Rolle der Massenmedien als zentraler gesellschaftlicher Vermittlungsinstanz (57). Daraus folgt für die Autoren jedoch nicht die Notwendigkeit einer kritischen Analyse der gesellschaftlichen Funktionsweise von Massenmedien in modernen Gesellschaften. Vielmehr nehmen sie die herausragende gesellschaftliche Definitionsmacht der Massenmedien zum Anlass, an die Verantwortung der führenden Eliten zu appellieren, die Entwicklung intermediärer Institutionen einer pluralistischen „civil society“ zu fördern (71). Auf die Unternehmenselite der Medienunternehmen beziehen Berger/Luckmann zwei von insgesamt drei Konzepten der soziologischen Elitetheorie. Zum einen verwenden sie das Konzept der Funktionselite, demzufolge moderne Gesellschaften in funktional differenzierte Subsysteme ausdifferenziert sind, die von den Experten in ihrem speziellen Fach geleitet werden. Darüber hinaus übernehmen die Medienführer die Aufgabe der sogenannten Wertelite:

„Im Begriff der Wertelite steckt die Vorstellung einer Krise der Werte. Weil der gesellschaftliche Zusammenhalt durch die Pluralität der Lebensstile und die Multiplizität der Lebenskreise als grundsätzlich gefährdet angesehen wird, braucht es eine davon unberührte Gruppe, die zumindest die ,letzten Werte“ des Großen und Ganzen verkörpert“" (Bude 2000: 10).

Mit ihrer Orientierung an ausgewählten Führungspersönlichkeiten folgen die Soziologen Berger/Luckmann dem programmatischen Selbstverständnis der Bertelsmann-Stiftung, die davon ausgeht, dass jene „für die Stiftungsarbeit notwendigen führungstechnischen Voraussetzungen zuallererst von Unterneh- 
merpersönlichkeiten erfüllt" werden können (Bertelsmann-Stiftung 1997: 10). Auffällig ist, dass das dritte Konzept soziologischer Elitetheorie, das der Machtelite, keine Anwendung findet. Dadurch geht ein konflikttheoretischer Gesellschaftsbegriff von vornherein verloren. Macht- und Herrschaftsstrukturen, deren Analyse einmal im Zentrum soziologischer Studien gestanden haben, verflüssigen sich im „modernen Pluralismus“ differenzierter Gesellschaften, folgt man Berger/Luckmann, in einem Brei der Medienpartizipation verschiedenster „moralischer Unternehmer“ (75), die die Medien der Massenkommunikation jeweils für ihre Zwecke einsetzen. Das Feld der Massenkommunikation erscheint bei ihnen als Jahrmarkt der Eitelkeiten, auf dem die unterschiedlichsten Lebensstile miteinander wetteifern. Der Aspekt einer demokratischen Legitimation, wie sie für öffentliche Medienanstalten - bei allen Einschränkungen - gelten, wird von Berger/Luckmann an keiner Stelle angesprochen. Es ist kaum anzunehmen, dass den Autoren soziale Statusunterschiede und daraus resultierende Zugangsbeschränkungen unbekannt sind, vielmehr drängt sich der Eindruck auf, wenn sie auf die besondere Rolle gesellschaftlicher Eliten hinweisen, dass eine hierarchische Strukturierung gesellschaftlicher Verhältnisse Berger/Luckmann zufolge mit einer pluralen Zivilgesellschaft vereinbar sei, ja von ihnen unhinterfragt vorausgesetzt wird. Offenkundig verbinden die Autoren mit dem Ausbau zivilgesellschaftlicher Strukturen keine traditionellen Formen demokratischer Partizipation. Das strategische Ziel des Projekts „Geistige Orientierung“ benennt hingegen Werner Weidenfeld, wenn er feststellt:

„Die vordringliche Aufgabe wird sein, dem Gedanken zum Durchbruch zu verhelfen, daß soziale Kohäsion die grundlegende Quelle der wirtschaftlichen Entwicklung und der ökologischen Sensibilität darstellt. Die kulturellen Grundlagen der Gesellschaft verdienen unsere volle Aufmerksamkeit als Basis für dauerhafte Entwicklung." (Weidenfeld 1997: 13)

Es geht mithin um die Verhinderung von Anomie durch die Gewährleistung des symbolisch (sinnhaft) vermittelten Zusammenhalts pluraler Gesellschaften. Bezüglich gesellschaftlicher Konfliktlinien und dadurch gefährdeter sozialer Kohäsion kommt Franz-Xaver Kaufmann in seinem für die Bertelsmann-Stiftung angefertigten Deutschlandbericht zu einer erstaunlich positiven Bilanz. Die in der Vergangenheit durch soziale Bewegung politisierten Konflikte im Umweltbereich und der Friedensinitiativen seien mittlerweile in routinierten und institutionalisierten Verfahrensweisen klein gearbeitet worden. Auf diese Weise sei eine „weitgehende Entdramatisierung und Konventionalisierung der Konflikte gelungen“ (Kaufmann 1997: 619). Einen potentiellen Konfliktherd hingegen vermutet Kaufmann im Wiederaufbrechen normativer Konflikte des älteren Typs. Die sich in den Debatten um den Standort Deutschland spiegelnden Verteilungskämpfe bergen seiner Ansicht nach möglicherweise sozialen Sprengstoff für zukünftige „Klassenkonflikte“ (ebd.: 189). Allerdings schätzt Kaufmann die Bedingungen einer normativen Dethematisierung des 
Klassenkonfliktes für die bundesrepublikanischen Verhältnisse als äußerst günstig ein (ebd.; 191). Für eine gesellschaftliche Institution wie die Bertelsmann-Stiftung, die ihre zentrale Aufgabe in der Schaffung sozialer Integration sieht, eröffnet sich damit offenbar ein wichtiges Arbeitsfeld.

\section{Fazit}

Wie lässt sich das Konzept der Bertelsmann-Stiftung abschließend charakterisieren? Dieter Plehwe und Bernhard Walpen haben jüngst am Beispiel der Mont Pèlerin Society eindrucksvoll aufgezeigt, wie marktradikale Think Tanks über den Zeitraum der letzten fünfzig Jahre zur Hegemoniegewinnung und -erhaltung neoliberaler Gesellschaftskonzepte beigetragen haben (Plehwe/Walpen 1999). Von diesen neoliberalen Denkfabriken, die sich, bei allen internen Differenzen, als ,closed shops ${ }^{6}$ verstehen, unterscheidet sich die Bertelsmann-Stiftung durch ihren nach allen gesellschaftlichen Seiten hin offenen Charakter. Indem sie versucht, verschiedene soziale Gruppen in ihre Reformstrategien einzubinden, verfolgt sie eine spezifische, neokorporatistische Strategie. Anders als noch in den 80er Jahren, als die Bertelsmann-Stiftung einen eher konfrontativen Kurs etwa gegenüber den Gewerkschaften eingeschlagen hatte ${ }^{10}$, zielt das neue Konzept auf ein weite Teile des gesellschaftlichen Spektrums integrierendes hegemoniales Projekt. Die strategische Orientierung der Bertelsmann-Stiftung mit ihrer einseitigen Ausrichtung an unternehmerischer Wettbewerbsfähigkeit kann als eine Art „Wettbewerbskorporatismus" (Rhodes 1998) bezeichnet werden, dem es gelingt, verschiedene gesellschaftliche Interessengruppen von dem Primat der ökonomischen Konkurrenz zu überzeugen. Insofern zeigen sich neben den formalen Unterschieden zwischen dem Neokorporatismus der Bertelsmann-Stiftung und den neoliberalen Konzepten marktkonformer Think Tanks auf der inhaltlichen Ebene deutliche Parallelen.

Begreift man das Projekt des Neoliberalismus als den Versuch einer weitgehenden Ökonomisierung des Politischen, dann entspricht die UnternehmensStiftung Bertelsmann sowohl in organisatorischer Hinsicht, wie auch in ihrem gesellschaftspolitischen Selbstverständnis den neoliberalen Think Tanks. Indem sie zum einen die Handlungsunfähigkeit des politischen Systems postuliert und den sozialen Innovationsprozess führenden Wirtschaftseliten überlassen möchte, während die „Bürger“ in eingehegten zivilgesellschaftlichen Instanzen mit Sinn versorgt und befriedet werden, vermittelt die Bertelsmann-

10 In seinem Beitrag Erfolg durch Partnerschaft (1986) wandte sich Reinhard Mohn noch gegen jede Art betrieblicher Mitbestimmung. Demgegenüber begreift er das deutsche Mitbestimmungsmodell in seinem neuen Buch als innovatives Instrument zum Zwecke sozialer Integration unter dem Gesichtspunkt einer harmonischen Unternehmensentwicklung (vgl. Mohn 2000). 
Stiftung über das Projekt „Geistige Orientierung“ ein einseitig wirtschaftskonformes Gesellschaftskonzept, in dem demokratische Verhältnisse allenfalls als über den Markt hergestellte Begegnungen gedacht werden. Kritikwürdig scheint mir neben der inhaltlichen Ausrichtung der Stiftung die spezifische Verzahnung von ökonomischer, organisationeller und politischer Macht zu sein. In Anbetracht dessen, dass die Stiftung von dem Mitinhaber des Bertelsmann Konzerns geleitet wird und 70\% der Kapitalanteile eines der weltweit größten Medienkonzerns mit staatlicher Hilfe am Fiskus vorbei schleust, um auf diese Weise die Unternehmenskontinuität zu gewährleisten, beeindruckt die Kaltschnäuzigkeit mit der es einer solchen Institution heute möglich ist, sich als „unabhängig“ sowohl von staatlicher Subventionierung wie auch von Unternehmensinteressen zu bezeichnen (vgl. Bertelsmann-Stiftung 1997: 18). Dass dies überhaupt möglich ist, erklärt sich wohl nur vor dem Hintergrund der heute vorherrschenden neoliberalen Hegemonie. Diese umfasst mit der Kapitulation von PolitikerInnen vor der Ideologie des Marktes und der Entpolitisierung der BürgerInnen zwei Entwicklungen, die eine zukünftige demokratische Gesellschaftsentwicklung gefährden.

Welche konkrete Utopie könnte demgegenüber all jene zusammenführen, die in Anbetracht der am Beispiel der Massenmedien beschriebenen gesellschaftlichen Machtkonzentrationen und den daraus folgenden Gefahren für demokratisch verfasste Gesellschaften nicht von der Absolutheit des Marktes überzeugt sind? Vielleicht findet sich ein Konsens, auf dem ein Projekt sich gründen muss, das dem Neoliberalismus begegnen will, in dem gemeinsamen Interesse an der Kunst, sich nicht dermaßen regieren zu lassen. Foucault (1992) hatte so sein Verständnis von Kritik umschrieben. Wenn der konservative Soziologe Vilfredo Pareto zum „Verfall der Eliten“ feststellte, dass diese in dem Moment verloren sind, wo sie nicht mehr an sich glauben, so gilt umgekehrt für die VertreterInnen eines demokratischen Gesellschaftsprojekts, Voraussetzung für eine erfolgversprechende Gegenstrategie ist die Überzeugung von der Notwendigkeit einer demokratisch motivierten Herrschaftskritik.

Am Beispiel des Bertelsmann Konzerns wird deutlich, dass ein solches Projekt nur noch im Sinne eines groß angelegten intellektuellen Verbunds denkbar ist, der zudem international orientiert sein muss. Eine solche Strategie der organisierten Mobilisierung von Intellektuellen auf der Grundlage moderner Kommunikationsmittel und mit dem Ziel einer „Internationale der Intellektuellen" verfolgt seit langem Pierre Bourdieu. Für einen Zusammenschluss von Intellektuellen mit dem Ziel einer kritischen Analyse gesellschaftlicher Machtstrukturen böten sich hier institutionelle Anknüpfungspunkte. Die vertiefte Untersuchung der Massenmedien könnte ein lohnendes Projekt darstellen, um der „List der imperialistischen Vernunft“ (Bourdieu/Wacquant 1998) zu begegnen. 


\section{Literatur}

Becker, Jörg 1985: Der Bertelsmann-Konzern, in: Peter H. Mettler (Hrsg.), Wohin expandieren multinationale Konzerne? Bd. 10, Arnoldshainer Schriften zur interdisziplinären Ökonomie, Frankfurt/M.

Bennhold, Martin 1999: Private Berater. Das Centrum für Hochschulentwicklung (CHE), der größte deutsche Medienkonzern und die Hochschulstrukturdebatte, in: Forum Wissenschaft, Heft 3, S. 51-56.

Berger, Peter L. (Hrsg.) 1997: Die Grenzen der Gemeinschaft. Konflikte und Vermittlung in pluralistischen Gesellschaften, Gütersloh.

Berger, Peter L. / Luckmann, Thomas 1995: Modernität, Pluralismus und Sinnkrise. Die Orientierung des modernen Menschen, Gütersloh.

- (1969): Die gesellschaftliche Konstruktion der Wirklichkeit. Eine Theorie der Wissenssoziologie, Frankfurt/M.

Bertelsmann-Stiftung (Hrsg.) 1997: Operative Stiftungsarbeit. Strategien - Instrumente - Perspektiven, Gütersloh.

Bertelsmann Stiftung/ Hans-Böckler-Stiftung (Hrsg.): Mitbestimmung und neue Unternehmenskulturen - Bericht der Kommission Mitbestimmung, Gütersloh 1998.

Dies. (Hrsg.) 2000: Vorteil Unternehmenskultur: Leitfaden für die Praxis, Gütersloh.

Bertram, Hans 1997: Familie leben. Neue Wege zur flexiblen Gestaltung von Lebenszeit, Arbeitszeit und Familienzeit, Gütersloh.

Bischoff, Joachim 2000: Internet-Wirtschaft oder Monopolherrschaft?, in: Sozialismus 2, S. 16-19.

Bourdieu, Pierre 1999: Ökologie der Kunst, in: die tageszeitung, 26. Oktober.

Bourdieu, Pierre / Wacquant, Loic 1998: Die List der Imperialistischen Vernunft, in: Liber, Heft 2, S. 3-21.

Briefs, Ulrich 2000: Mythos 'Informationsgesellschaft', in: Z. Zeitschrift Marxistische Erneuerung, Nr. 41, S. 81- 93.

Bude, Heinz 2000: Auf der Such nach Eliten, in: Kursbuch, Die neuen Eliten, Heft 139, Berlin, S. 9-16.

Bundesmann-Jansen, Jörg/ Pekruhl, Ulrich 1992: Der Medienkonzern Bertelsmann - Neues Management und gewerkschaftliche Betriebspolitik, HBS-Forschung, Band 7.

Bundesverband Deutscher Stiftungen 2000: Verzeichnis deutscher Stiftungen, Darmstadt.

Deppe, Frank 2000: Sozialpartnerschaft ohne Alternative? Anmerkungen zur neueren Debatte um die industriellen Beziehungen, in: U. Klitzke/ H. Betz/ M. Möreke (Hrsg.): Vom Klassenkampf zum Co-Management? Perspektiven gewerkschaftlicher Betriebspolitik, Hamburg, 179-213.

Dettling, Warnfried 1995: Politik und Lebenswelt. Vom Wohlfahrtsstaat zur Wohlfahrtsgesellschaft, Gütersloh.

Fischer, Frank 1996: Die Agenda der Elite. Amerikanische Think Tanks und die Strategie der Politikberatung, in: PROKLA 104, S. 463-481.

Foucault, Michel 1992: Was ist Kritik?, Berlin.

Gellner, Winand 1995: Ideenagenturen für Politik und Öffentlichkeit. Think Tanks in den USA und Deutschland, Opladen/Wiesbaden.

Geschäftsbericht 1998/99 der Bertelsmann AG, Gütersloh.

Greiffenhagen, Martin 1997: Politische Legitimität in Deutschland, Gütersloh.

Hachmeister, Lutz/ Rager, Günter 1997: Wer beherrscht die Medien? Die 50 größten Medienkonzerne der Welt, München.

Hautsch, Gert 1999: Zwischen Buch und Börse. Die deutschen Medienbranche zwischen Sommer 1998 und Sommer 1999, in: Reader Medienökonomie 1999, S.1-35.

Hoffmann-Riem, Wolfgang 1995: Aufgaben zukünftiger Medienregulierung, in: Ders./ Simonis, Heide (Hrsg.): Chancen Risiken und Regelungsbedarf im Übergang zum Multi-MediaZeitalter. Dokumentation zum Medienworkshop am 21. August 1995, Kiel, S. 14-32.

Ders. 2000: Politiker in den Fesseln der Mediengesellschaft, in: Politische Vierteljahresschrift, Heft 1, S. 107-127.

Horizont 30, 1999: Die größten deutschen Medienunternehmen.

Huber, Wolfgang 1998: Kirche in der Zeitenwende. Gesellschaftlicher Wandel und Erneuerung der Kirche, Gütersloh. 
Jäckel, Michael/ Winterhoff-Spurk, Peter (Hrsg.) 1996: Mediale Klassengesellschaft? Politische und soziale Folgen der Medienentwicklung, München.

Jahresbericht der Hans-Böckler-Stiftung 1998, Düsseldorf.

Jochum, Michael 1999: Worte als Taten. Der Bundespräsident im demokratischen Prozess der Bundesrepublik Deutschland, Gütersloh.

Kannapin, Detlef 1995: Medien, Ideologie, Herrschaft, in: Z. Zeitschrift Marxistische Erneuerung, Nr. 23, S. 57-66.

Kaufmann, Franz-Xaver 1997: Normative Konflikte in Deutschland: Basiskonsens, Wertewandel und soziale Bewegung, in: Peter L. Berger a.a.O., S. 155-197.

Keupp, Heiner 1992: Verunsicherungen. Risiken und Chancen des Subjekts in der Postmoderne, in: Thomas Rauschenbach/ Hans Gängler (Hrsg.): Kultur und Alltag. Soziale Welt, Sonderband 6, S. 325-340, Göttingen.

Kommission Mitbestimmung 1998: Mitbestimmung und neue Unternehmenskulturen - Bilanz und Perspektiven. Bericht der Kommission Mitbestimmung. Bertelsmann Stiftung und HansBöckler-Stiftung, Gütersloh.

Kresse, Herrmann 1995: Pluralismus, Markt und Medienkonzentration - Positionen, Opladen/ Wiesbaden.

Kursbuch, Die neuen Eliten, Heft 139, Berlin.

Luckmann, Thomas (Hrsg.) 1998: Moral im Alltag. Sinnvermittlung und moralische Kommunikation in intermediären Institutionen, Gütersloh.

Maresch, Rudolf 1995: Mediatisierte Öffentlichkeit, in: Leviathan, Heft 3, S. 394-416.

Martens, Helmut 1999: Auslaufmodell oder Reformkonzept für die moderne Teilhabe- gesellschaft? Eine kritische Würdigung der Ergebnisse der neuen Mitbestimmungskommission von Hans-Böckler-Stiftung und Bertelsmann-Stiftung, in: Soziale Welt, Heft 1, S. 67-85.

Memorandum des Initiativkreis Bildung der Bertelsmann Stiftung unter Schirmherrschaft des Bundespräsidenten 1999: Zukunft gewinnen, Bildung erneuern, Gütersloh.

Mohn, Reinhard 1986: Erfolg durch Partnerschaft, Berlin.

Ders. 1992: Neue Ziele in der Welt der Arbeit, Gütersloh.

Ders. 1992a: Sozialpolitik in Europa, Gütersloh.

Ders. 1993: Effizienz und Evolutionsfähigkeit im öffentlichen Dienst, Gütersloh.

Ders. 1995: Freiheit für den kreativen Menschen, Gütersloh.

Ders. 1996: Unternehmenskultur als Bedingung für unternehmerischen Erfolg, Gütersloh.

Ders. 1996a: Gemeinschaftsfähigkeit - als Brücke zwischen dem einzelnen und der Gesellschaft, Gütersloh.

Ders. 1996b: „Ein Segen, daß uns das Geld ausgeht“. Interview mit Reinhard Mohn, Gütersloh.

Ders. 1996c: Ziele einer operativen Stiftung, Gütersloh.

Ders. 1997: Deutschland im Wettbewerb der Ordnungssysteme, Gütersloh.

Ders. 1997a: Geistige Orientierung als Grundlage der Gemeinschaftsfähigkeit, Gütersloh.

Ders. 1997b: Unternehmerische Führung im Großbetrieb, Gütersloh.

Ders. 1998: Kooperation in Wirtschaft und Gesellschaft, Gütersloh.

Ders. 1999, in: Zeitzeugen des Jahrhunderts, Phoenix am 28. November.

Ders. 2000: Menschlichkeit gewinnt. Eine Strategie für Fortschritt und Führungsfähigkeit, Gütersloh.

Müller, Hans-Peter 1992: Sozialstruktur und Lebensstile, Frankfurt/M.

Nielandt, Jörg/ Brodkorb Mathias 2000: Ausbau der Zivilgesellschaft. Non-Profit-Organisationen und Dritter Sektor, in: Sozialismus Supplement, Heft 9, Hamburg.

Plehwe, Dieter/ Walpen, Bernhard 1999: Wissenschaftliche und wissenschaftspolitische Produktionsweisen im Neoliberalismus. Beiträge der Mont Pèlerin Society und marktradikaler Think Tanks zur Hegemoniegewinnung und -erhaltung, in: PROKLA, Heft 115, S. 203-235.

Prokop, Dieter 1995: Medien-Macht und Massen-Wirkung. Ein geschichtlicher Überblick, Freiburg im Breisgau.

Reader Medienökonomie 1999. Materialien zur wirtschaftlichen Situation, zu den Konzentrationsprozessen und zur Beschäftigungsentwicklung im Verlagsbereich, hrsg. v. Branchenarbeitskreis Verlage der IGM, HBV, DAG, Stuttgart/ Düsseldorf/ Hamburg.

Reinicke, Wolfgang H. 1996, Lotsendienste für die Politik. Think Tanks - amerikanische Erfahrungen und Perspektiven für Deutschland, Gütersloh. 
Rhodes, Martin 1998: Globalization, Labor Markets and Welfare States: A Future of 'Competitive Corporatism'?, in: M. Rhodes/ Y. Mény (Hrsg.): The Future of European Welfare: A New Social Contract? London, S. 178-203.

Röper, Horst 1999: Formationen deutscher Medienmultis 1998/1999, in: Media Perspektiven 7, S. 345- 378.

Salomon, Lester M./ Anheier, Helmut K. 2000: Der dritte Sektor. Aktuelle internationale Trends, Gütersloh.

Schatz, Heribert/ Jarren, Otfried/ Knaup, Bettina 1998: Machtkonzentration in der Multimediagesellschaft? Beiträge zu einer Neubestimmung des Verhältnisses von Politischer und Medialer Macht, Opladen.

Schmidtchen, Gerhard 1996: Lebenssinn und Arbeitswelt. Orientieruing im Unternehmen, Gütersloh.

Stone, Diane 1996: Capturing the Political Imagination. Think Tanks and the Policy Process, London-Portland, OR.

Tätigkeitsbericht 98/99 der Bertelsmann-Stiftung 1999: Gütersloh.

Verlag Bertelsmann-Stiftung 1999/2000, Gütersloh.

Wehner, Ewald 1998: Umwidmung der Mitbestimmung, in: Sozialismus, Heft 10, S. 44-46.

- 1999: Mitbestimmung oder Mitgestaltung? Die Auseinandersetzung um ein neues Betriebsverfassungsgesetz und die Rolle der Hans-Böckler-Stiftung, in: Sozialismus, Heft 10, S. 25- 27.

Weidenfeld, Werner 1997: Vorwort, in: Peter L. Berger (Hrsg.): a.a.O., S. 11-15.

Ders./ Rumberg, Dirk 1994: Orientierungsverlust - Zur Bindungskrise der modernen Gesellschaft, Gütersloh.

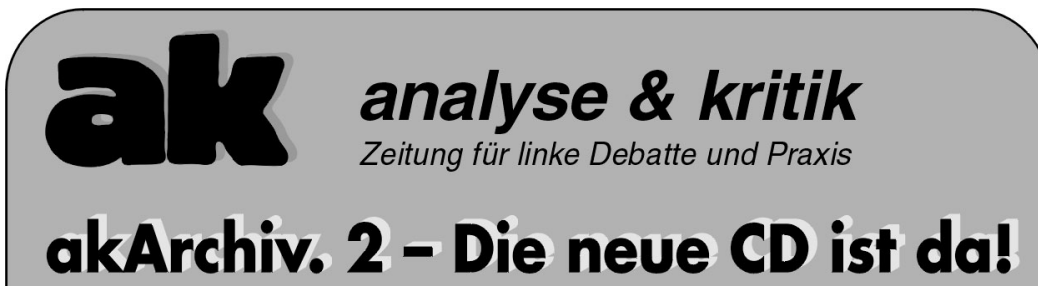

Jetzt abonnieren: Wer ein Jahresabo bestellt oder vermittelt, bekommt die aktuelle CD akArchiv. 2 geschenkt. Sie enthält fast 5.000 Artikel der Jahrgänge von 1992 bis 10/2000. Die CD kostet 69 DM (für ak-Abonnentlnnen 49 DM und 20 DM als update der ersten CD akArchiv.) + jeweils 5 DM Porto

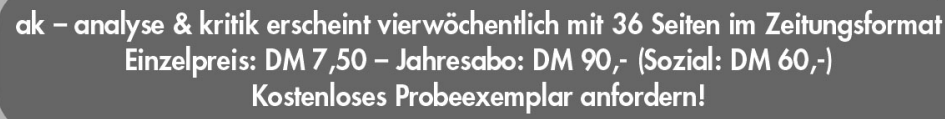

ak - analyse \& kritik · Rombergstraße 10 - 20255 Hamburg · www.akweb.de Tel.: 040-40170174 • Fax: $40170175 \cdot$ E-mail: ak-Redaktion@cl-hh.comlink.de 